\title{
Zahnpflege beugt Prädiabetes vor
}

\author{
Periodontale Bakterien begünstigen sehr deutlich die Entstehung eines Prädiabetes. Für Haus- \\ ärzte ist das ein weiterer Grund, auch auf die Mund- und Zahnpflege der Patienten zu achten.
}

Dass eine Periodontitis das Risiko für Typ-2-Diabetes erhöht, ist bekannt. Eine Studie mit 300 Nicht-Diabetikern zwischen 20 und 55 Jahren untersuchte nun den Zusammenhang mit der Vorstufe Prädiabetes. Bei $18 \%$ der Patienten lag gemäß den internationalen Klassifikationen ein Prädiabetes vor.

Bei 58\% der Probanden stellten die Forscher eine mittlere bis schwere Periodontitis fest. Für diese Gruppe errechnete sich eine 1,74-fach erhöhte Prävalenz von Prädiabetes. Von den 11 identifizierten Bakterienspezies waren einige besonders gut als Prädiktoren für Prädiabetes geeignet. Bei Vorliegen von Porphyromonas gingivalis war die Prävalenz um den Faktor 3,41 erhöht, bei Aggregatibacter actinomycetemcomitans um 2,48 und bei Treponema denticola um 1,99 .

Insgesamt war ein höheres Kolonisationslevel für spezifische periodontale
Mikrobiota bei nicht diabetischen Erwachsenen direkt mit einer höheren Prävalenz für Prädiabetes assoziiert. Für die Autoren unterstreicht das den Stellenwert von Zahnschutz und Zahnpflege in der öffentlichen Gesundheitsvorsorge. Immerhin sind periodontale Bakterien auch an der Entstehung kardiovaskulärer und anderer chronischer Erkrankungen beteiligt.

- Demmer RT, Jacobs DR Jr, Singh R et al. Periodontal Bacteria and Prediabetes Prevalence in ORIGINS: The Oral Infections, Glucose Intolerance, and Insulin Resistance Study. J Dent Res. 2015;94:2015-115

\section{KOMMENTAR}

Zähneputzen beugt erhöhtem Diabetesrisiko vor - das ist das einfache Fazit der Studie. Die Ergebnisse sind beeindruckend. Bestimmte periodontale Bakterien erhöhen das Prädiabetesrisiko so stark wie ein BMI von 33. Letzterer ist gar nicht so leicht loszuwerden. Zähneputzen ist da viel einfacher. Die Autoren zeigen, dass die meisten Mundbakterien das
Prädiabetesrisiko zwischen 2- und 3,5-fach erhöhen und nur wenige einen geringen oder, zumindest auf dem Papier, sogar "protektiven" Einfluss haben. Auch wir als Nichtzahnärzte sollten überlegen, was das für unsere Arbeit bedeutet.

Zähneputzen ist sicher der erste Schritt, löst aber nicht alle mikrobiellen Probleme im Mund - vor allem, wenn tiefe Zahnfleischtaschen vorliegen. Antimikrobielle Mundwasser sind meines Erachtens sehr wenig untersucht, und es wird eher davon abgeraten. Sollten wir da nicht viel stärker auch einen Blick auf den Zahnstatus des Patienten lenken und ihn zum Zahnarzt schicken? Wenn die Ergebnisse dieser Studie stimmen, ist der Einfluss der Bakterienflora im Mund auf das Entstehen eines Diabetes und auf den Behandlungserfolg sehr groß. Wir sollten immer im Kopf haben, dass man mit guter Zahnhygiene den Diabetes in großen Teilen wegputzen kann.

Prof. Dr. med. P. Schwarz

\section{Ist dieses Baby ein Mädchen oder ein Junge?}

\begin{abstract}
Bei einem reifen Neugeborenen stellte man unbestimmte Genitalien fest (Abb. A). Die Familienanamnese bezüglich genetischer Erkrankungen war unauffällig. Das Neugeborene hatte eine Sinustachykardie mit einer Herzfrequenz von 179/min ohne Hypotonie. Im Labor fielen eine Hypokaliämie von 7,4 $\mathrm{mmol} / \mathrm{l}$, eine Hyponatriämie mit $125 \mathrm{mmol} / \mathrm{l}$, eine erhöhte 17-Hydroxyprogesteron-Konzentrationen von $196 \mu \mathrm{g} / \mathrm{l}$ (Normalbereich: $0,5-2,4)$ sowie Testosteronspiegel von über 15 $\mathrm{ng} / \mathrm{ml}$ (Normalbereich: 0,1-0,8) auf. Die Oberbauch-Sonografie zeigte beidseits vergrößerten Nebennieren. Hoden waren nicht auffindbar, und die Chromosomenanalyse ergab einen Karyotyp 46 XX. Man stellte die Diagnose eines Salzverlustsyndroms bei beidseitiger kongenitaler adrenaler Hyperplasie infolge eines 21-Hydroxylase-Mangels bei einem weiblichen Neugeborenen.
\end{abstract}
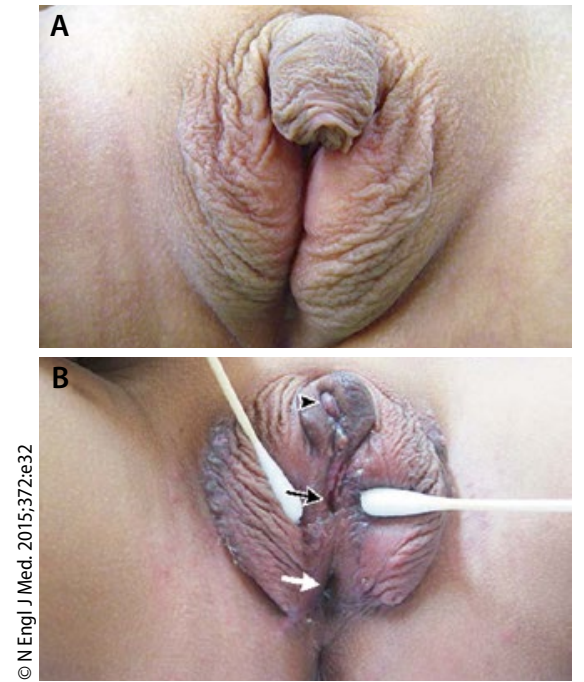

Unbestimmte Genitalien (A), chirurgische Rekonstruktion (B).
Unbestimmte Genitalien sind charakterisiert durch eine Hypertrophie der Klitoris und der Labia majora und dem Vorhandensein eines urogenitalen Sinus, der an eine Hypospadie mit Kryptorchismus bei einem männlichen Neugeborenen erinnert.

Man begann eine Infusionstherapie zum Ausgleich der Elektrolytveränderungen und substituierte Gluko- und Mineralokortikoide. Im Alter von zwei Jahren wurde eine aufwändige chirurgische Rekonstruktion mit Resektion des Urogenitalsinus (Abb. B, schwarzer Pfeil), einer Klitoroplastik (Pfeilspitze) und einer Vaginoplastie (weißer Pfeil) vorgenommen. Unter fortlaufender Substitution von Kortikoiden entwickelte sich das Kind normal.

Prof. Dr. med. H. S. FüeßI

- Ko PJ, Yeh ML (ed103434@edah.org.tw). Congenital adrenal hyperplasia. N Engl J Med. 2015;372:e32 